\title{
Immune Escape Mechanisms and their Clinical Relevance in Head and Neck Squamous
} Cell Carcinoma

*Barbara Seliger ${ }^{1,2}$, Chiara Massa ${ }^{1}$, Bo Yang ${ }^{1}$, Daniel Bethmann ${ }^{3}$, Matthias Kappler ${ }^{4}$, Alexander Eckert $^{4,5}$, Claudia Wickenhauser ${ }^{3}$

${ }^{1}$ Institute of Medical Immunology, Martin Luther University Halle-Wittenberg, 06112 Halle, Germany

${ }^{2}$ Fraunhofer Institute of Cell Therapy and Immunology, 04103 Leipzig, Germany

${ }^{3}$ Institute of Pathology, Martin Luther University Halle-Wittenberg, 06112 Halle, Germany

${ }^{4}$ Department of Oral and Maxillofacial Plastic Surgery, Martin Luther University Halle-Wittenberg, 06120 Halle (Saale), Germany

${ }^{5}$ Klinik für Mund-, Kiefer- und Plastische Gesichtschirurgie, Universitätsklinik der Paracelsus Medizinischen Privatuniversität; 90471 Nürnberg, Germany

*Corresponding author:

\author{
Prof. Dr. Barbara Seliger \\ Martin Luther University Halle-Wittenberg \\ Institute of Medical Immunology \\ Magdeburger Str. 2 \\ 06112 Halle (Saale) \\ Germany
}

Telephone:

(+49) (345) 557 - 4054

Fax:

$(+49)(345) 557-4055$

E-mail:

barbara.seliger@uk-halle.de

Keywords: head and neck squamous cell carcinoma; immune escape; tumor microenvironment; immune responses; immunotherapy 


\section{Abbreviations}

$\beta_{2}$-m, $\beta_{2}$-microglobulin; CAF, cancer associated fibroblast; CSC, cancer stem cell; CTL, cytotoxic T lymphocyte; DC, dendritic cell; ECM, extracellular matrix; EGF-R, epidermal growth factor receptor; ER, endoplasmic reticulum; FDA, Food and Drug Administration; HLA, human leukocyte antigen; HNSCC, head and neck squamous cell carcinoma; HPV, human papilloma virus; ICP immune checkpoint; ICPi, immune checkpoint inhibitor; IFN, interferon; LMP, low molecular weight protein; mAb, monoclonal antibody; MDSC, myeloid-derived suppressor cell; mTOR, mammalian target of rapamycin; MSI, multispectral imaging; NK, natural killer; OS, overall survival; PBL, peripheral blood lymphocytes; PBMNC, peripheral blood mononuclear cells; PD1, programmed death receptor 1; PD-L1, programmed death ligand 1; PFS, progression-free survival; PI3K, phosphatidyl-linositol-3-kinase; R/M, recurrence and or metastatic; STAT, signal transducer and activator of transcription; TAA, tumor-associated antigen; TAM, tumor associated macrophages; TAP, transporter associated with antigen processing; TCR, T cell receptor; TIL, tumor-infiltrating lymphocyte; TLS, tertiary lymphoid structure; TME, tumor microenvironment; Treg, regulatory T cell; TSA, tumor-specific antigen; VEGF, vascular endothelial growth factor; VEGF-R, vascular endothelial growth factor receptor. 


\begin{abstract}
Immunotherapy has been recently approved for the treatment of relapsed and metastatic head and neck squamous cell carcinoma (HNSCC). However, the response of patients is limited and the overall survival remains short with a low rate of long-term survivors. There exists growing evidence that immune escape mechanisms play an important role for the low efficacy of immunotherapies in this disease. These are caused by diverse complex processes characterized by (i) changes in the expression of immune modulatory factors in tumour cells, (ii) alterations in the frequency and composition of immune cell subpopulations in the tumour microenvironment and peripheral blood leading to reduced innate and adaptive immune responses, (iii) impaired homing of immune cells to the tumour site as well as (iv) the presence of immune suppressive soluble and physical factors in the tumour microenvironment. We here summarize the major immune escape strategies of HNSCC lesions, the role of the tumor microenvironment in this process, the clinical relevance of HNSCC-induced immune tolerance, currently employed immunotherapeutic approaches and possibilities to overcome resistance to immunotherapy thereby improving the HNSCC patients' survival.
\end{abstract}




\section{Introduction}

Head and neck squamous cell carcinoma (HNSCC) represents the sixth most common cancer worldwide with 700.000 new cases diagnosed in 2019 with a mortality rate ranging between $40-$ $50 \%$ [1] [2].The development of HNSCC has been correlated to life-style risk factors like smoking and alcohol consume as well as to infections with human papilloma viruses (HPV) [3] [4]. Conventional treatment for HNSCC comprises of surgery, radio- and/or chemotherapy, which is associated with a substantial high morbidity and toxicity rate. Therefore, more effective therapies for the treatment of this disease are urgently needed. Recently, a number of targeted therapies using either monoclonal antibodies or tyrosine kinase inhibitors directed against the epidermal growth factor receptor (EGF-R), the vascular endothelial growth factor (VEGF) and its receptor (VEGF-R), the phosphatidyl-inositol-3-kinase (PI3K) and the mammalian target of rapamycin (mTOR) has been approved for the treatment of HNSCC, such as e.g. cetuximab, panitumumab, zalutumumab, gefitinib, erlotinib, lapatinib, bevacizumab, sorafenib, rapamycin and everolimus, with significant, but still limited response rates ranging between $10-15 \%$. The low efficacy of these targeted therapies might depend on the molecular and immunological make up as well as the heterogeneity of HNSCC lesions with a number of genetic drivers of oncogenesis altering also the anti-tumoral immune responses. During the last years, immunotherapy approaches have been shown to improve the survival of HNSCC patients, which include immunomodulatory antibodies, vaccines, oncolytic viruses and adoptive cell therapy. Treatment with immune checkpoint inhibitors ( $\mathrm{iCPi}$ ) targeting the programmed death receptor 1 (PD-1), like nivolumab and pembrolizumab and its ligand PD-L1 (atezolizumab), had significant clinical effects in a subset of HNSCC patients. However, the majority of HNSCC patients are resistant to these immunotherapies. This might be due to HNSCC (stem) cell mediated progression, recurrence or metastases, but the underlying molecular mechanisms of resistances are so far mainly unknown [5-7]. It is currently being discussed whether cellular and soluble factors of the tumor microenvironment (TME), alterations of the immunogenicity of tumor cells including cancer stem cells (CSC) per se, the composition of peripheral blood mononuclear cells (PBMNC) and/or the presence of immune suppressive mediators might lead to immune evasion.

Approximately 50 years ago, research suggested that pre-malignant cells frequently occur, but are spontaneously eliminated by the immune system, before an invasive tumor can develop thereby preventing neoplastic transformation. This hypothesis was supported by epidemiological data demonstrating that patients infected with the human immunodeficiency virus and patients receiving immune suppressive therapy were more prone to cancer [8] [9]. Furthermore, tumor patients can develop spontaneous cancer regressions [10] as well as an increase of HNSCC 
incidence was detected in transplanted as well as immune suppressed patients [11]. These data led to the assumption that tumors can evade immune responses by developing diverse immune escape strategies, which will allow the selection for and escape of non-immunogenic cells from immune surveillance by inhibiting their cytotoxic functions of immune cells via distinct mechanisms [12]. Thus, there exists an interplay between the immune system and tumors, which was defined as cancer immunoediting [13-16]. Next to the direct immune escape mechanisms of tumors, an immune suppressive network within the TME was described. This observation represents the basis for the development of novel individualized immunotherapies for cancer. In this review, the diverse processes leading to tumor immune escape in HNSCC and their impact on immunotherapies will be discussed.

\section{$\underline{\text { Immune escape mechanisms of tumors }}$}

It is generally accepted that an effective $\mathrm{T}$ cell activation is required for a proper anti-tumor response [17], which consists of two distinct signals. The first signal is mediated by the interaction of the $T$ cell receptor (TCR) with tumor antigens presented by human leukocyte antigens (HLA) class I molecules, the second by the interaction of co-stimulatory receptors with their ligands resulting in T cell proliferation, cytokine secretion and cytotoxicity. A pre-existing intratumoral antitumor immune response has been associated with a favourable outcome and responsiveness to immunotherapies [18].

Tumor antigens can be classified into tumor-associated antigens (TAAs) or tumor-specific antigens (TSA) [19] [20]. TAAs are expressed by both malignant and healthy cells, while TSA are expressed only by tumor cells. TSA and TAA are proteins, from which T cell epitopes are generated by the multicatalytic proteasome complex consisting of the constitutive subunits $X, Y$ and $Z$ as well as the interferon (IFN) inducible low molecular weight proteins (LMP) 2, 7 and 10. The yielded peptides are transported via the transporter associated with antigen processing (TAP) submits TAP1 and TAP2 from the cytosol into the endoplasmic reticulum (ER) and then loaded onto HLA class I molecules. The trimeric complex consisting of the MHC class I heavy chain $(\mathrm{HC})$, $\beta_{2}$-microglobulin $\left(\beta_{2}-\mathrm{m}\right)$ and the respective TAA/TSA derived epitope is then shuttled via the transGolgi to the cell surface and presented to $C D 8^{+}$cytotoxic T lymphocytes (CTL) [21] [22]. Complete loss or downregulation of HLA class I surface expression leads to evasion of tumor cells from CTL recognition. The loss or downregulation of HLA class I expression is often mediated by an abnormal expression and function of major components of the HLA class I antigen processing machinery (APM). This can be either due to structural abnormalities or due to deregulation at the 
transcriptional, epigenetic, post-transcriptional as well as post-translational level of APM components. In addition, the loss of adhesion molecules, e.g. ICAM-1, and the frequency of tumor infiltrating lymphocytes (TILs) interferes with anti-tumoral responses [23, 24]. Furthermore, nonclassical HLA class I antigens, like HLA-G and HLA-E, may be expressed in tumors at a high frequency leading to tolerance not only against CTL, but also against natural killer (NK) cellmediated cytotoxicity. In addition, immune suppressive ligands, like PD-L1 and nectin 4, and apoptosis-inducing ligands, such as the fas ligand and TRAIL, have been reported to be expressed by tumor cells and/or host's myeloid cells, which results in apoptosis or anergy of T and/or NK cells.

The composition and frequency of immune cells within the TME and peripheral blood plays an important role in tumorigenesis. While NK cells, $\mathrm{CD} 4^{+}$and $\mathrm{CD} 8^{+} \mathrm{T}$ cells, dendritic cells (DC) and proinflammatory M1 macrophages promote anti-tumor immune responses, heterogeneous populations of myeloid-derived suppressor cells (MDSC), FOXP3 ${ }^{+}$regulatory $\mathrm{T}$ cells and $\mathrm{M} 2$ macrophages counteract tumor immunity [17] [25]. The complexity of the immune system is reflected by the composition of the immune cell infiltrate, the amount of inflammation and the frequency of TILs, which affect the overall survival (OS) of patients. These observations resulted in the categorization of inflamed, immune desert and immune excluded tumor phenotypes [26]. Tumors may also secrete various immune suppressive and anti-apoptotic factors like TGF- $\beta$, IL10, PGE2 and IL-6 as well as release immune suppressive extracellular vesicles (EV), such as micro-vesicles and exosomes and different metabolites including arginase, adenosine, indolamin2,3-dioxygenase (IDO) and nitric oxygen species (NOS) [27][28]. Furthermore, the immune system is also affected by metabolic changes in the TME due to hypoxic and acidic conditions [29].

The role of classical and non-classical HLA class I and antigen presentation and processing in HNSCC immunotherapies in HNSCC

The expression of classical HLA class I antigens was shown to be diminished at a high frequency varying from 20 up to $80 \%$ of HNSCC lesions and cell lines whereas $\beta_{2}$-m expression was absent in approximately $16 \%$ of tumors [30] [31] [32] [33]. There was no difference in the frequency of HLA class I abnormalities between HPV- and $\mathrm{HPV}^{+}$tumors [34]. Interestingly, the loss or downregulation of HLA class I and $\beta_{2}$-m expression was correlated with the loss of chromosomal regions at $6 \mathrm{p} 21.3$ and loss of heterozygosity $(\mathrm{LOH})$ at $6 \mathrm{p} 21.3$ and $15 \mathrm{q}[32,35]$. 
Reduced expression of HLA class I antigens was associated with a downregulation in the expression of various APM components. While TAP1, LMP2, LMP7 and $\beta_{2}-m$ expression were downregulated in up to $70 \%$ of HNSCC lesions, calnexin and ERp57 were only downregulated in approximately $25 \%$ of lesions [33, 36, 37]. The extent of HLA class I APM defects was associated with a low $\mathrm{CD}^{+} \mathrm{T}$ cell infiltration. It has been also demonstrated that overexpression and activation of epidermal growth factor receptor (EGF-R), which is expressed at a high frequency in HNSCC specimens, can lead to a downregulation of HLA class I by affecting downstream pathways, such as STAT1, MAPK and PI3K/AKT. This impaired HLA class I expression could be reversed by treatment with respective inhibitors or by IFN- $\gamma$ [38]. In contrast, HLA class II expression was only found in a minority of HNSCC lesions, but at a high frequency in stromal cells. Interestingly, HLA class II expression was higher in $\mathrm{HPV}^{+}$compared to HPV- tumors [39].

Next to classical HLA class I antigens, the non-classical HLA-G expression was detected in more than $50 \%$ of HNSCC lesions, while healthy controls lacked HLA-G expression [40] [41] [32] [33] [37]. A higher frequency of HLA-G-expression was found in $\mathrm{HPV}^{+} \mathrm{HNSCC}$ when compared to HPV- HNSCC [42]. Furthermore, significantly higher HLA-G expression levels were detected in metastases compared to primary tumors [43]. HLA-E was recently also found to be expressed on HNSCC [44]. Regarding PD-L1 expression, its frequency highly varies in the HNSCC tumors depending on the intratumoral localization and the tumor specification as PD-L1 expression was higher in the invasive tumor margin than in the tumor center and higher in $\mathrm{HPV}^{+}$than in $\mathrm{HPV}^{-}$ HNSCC [34]. As for HLA-G, PD-L1 expression was more frequent in metastases than in primary tumors [45]. Interestingly, the metabolic tumor volume and the glycolytic activity correlated significantly positive with PD-L1 expression [46].

\section{$\underline{\text { Tumor microenvironment and HNSCC }}$}

A number of soluble and cellular factors within the TME of HNSSC have been described to be involved in the immune escape of this disease [47]. Furthermore, the TME has the ability to adapt to environmental demand depending on the tumor metabolism and hypoxia. This reprogramming not only determined the fate and functions of tumor cells, but also of immune cells. Both factors are known to play key roles in the course of HNSCC and to support the progression of this disease [48]. Indeed, due to hypoxia and acidic conditions HNSCC switch to the glycolytic metabolism, which further reduces the $\mathrm{pH}$, which is mediated by the production of lactic acid [49][50]. Importantly, these metabolic alterations in the TME not only affect the HNSCC lesions by altering 
the expression of immune modulatory molecules, such as e.g. an increased expression of PD$\mathrm{L} 1$, but also change the immune cell repertoire and activity. This leads to a reduced $\mathrm{T}$ cell activation, proliferation, cytotoxicity and diminished antigen processing of DC with simultaneous attraction and accumulation of immune suppressive cells (Treg, MDSC and TAMs) [51] [52]. This is accompanied by a high frequency of $\mathrm{CD} 4^{+}$Tregs, which was associated with an increased expression of TNF receptor family members, but a reduced expression of genes involved in the IFN pathway. Interestingly, OX40, PD-1 and CTLA were enriched in T cells isolated from HNSCC [53]. MDSC were found to be recruited in HNSCC, but their role in this disease has not yet been analysed in detail. Moreover, the hypoxic condition induces a stromal accumulation of TAMs, which are responsible for the epithelial mesenchymal transition (EMT) in this disease and might represent an immunotherapeutic target for HNSCC patients [54]. Furthermore, the hypoxiainduced release of VEGF and various chemokines could induce monocytes to differentiate into TAMs, while the secreted VEGF can also cause an abnormal angiogenesis [55] [56]. This angiogenic switch further contributes to the maintenance of the hypoxic TME, additionally lowering the tissue $\mathrm{pH}$.

Last but not least, remodelling of the extracellular matrix (ECM) mediated by cancer-associated fibroblasts (CAFs) occurs and leads to the protection of tumor cells by a biophysical barrier to effector $\mathrm{T}$ cells and to resistance against monoclonal antibodies (mAbs) targeting EGF-R [57]. Interestingly, late stage HNSCC consists of more than $80 \%$ CAFs, which secrete HGF thereby inducing a glycolytic switch in tumor lesions [58]. Furthermore, CAFs promote an immune suppressive TME through the induction of a pro-tumoral phenotype in macrophages [46]. This further reflects that different immune escape mechanisms are dependent of each other and crossover thereby creating a redundant and highly efficient immune suppressive system.

Recently, the immune landscape of HNSCC was analysed and genetic profiles were identified, which delineate tumors in immune active and immune exhausted phenotypes [59]. This gives information on the immune status of HNSCC patients, which could be used for the development of novel (immuno)therapies [60]. Combination of single cell RNA sequencing (scRNA-seq) with multispectral imaging (MSI) that allow to characterize the spatial localization of immune cells and their cellular neighbourhood within the TME will give deeper insights into the immune cell repertoire of $\mathrm{HNSCC}$, their transcriptional states and differentiation trajectories as well as into the cellular crosstalk in the TME with potential relevance to tumor progression [61-63].

In depth analyses of the composition of innate and adaptive immune cells in the TME of patients in $\mathrm{HPV}^{-}$as well as $\mathrm{HPV}^{+} \mathrm{HNSCC}^{-}$lesions highlighted differences in the immune cell repertoire in 
dependence of the HPV status of the tumor specimen, which might have also implications for their treatment with immunotherapies. The distinct immune cell repertoire in the TME might be associated with viral antigens leading to the activation of primarily innate immune responses followed by adaptive immune responses mediated by $T$ and $B$ lymphocytes. Furthermore, single cell analyses demonstrated subpopulations of immune cells in particular of exhausted $\mathrm{CD} 8^{+} \mathrm{T}$ cells with different biological functions [64].

\section{Correlation of the efficiency of (immuno)therapies with immune escape mechanisms}

It has been demonstrated that established therapies for HNSCC patients, such as radiotherapy, chemotherapy as well as mAbs directed against the EGF-R affect the TME. Since CD8 ${ }^{+}$TILs exhibit a high frequency of PD1 and TIM-3 expression accompanied by high levels of granzymes and perforin and is associated with the clinical outcome, a regulatory role for TIM-3 and PD1 was suggested in cetuximab-promoting cytolytic activities of $\mathrm{CD}^{+}$TILs. Furthermore, the increased frequency of PD $-1^{+}$and $\mathrm{TIM}-3^{+} \mathrm{CD} 8^{+}$TILs was inversely correlated with the clinical outcome of cetuximab therapy [65].

Treatment of HNSCC patients with immunotherapies increases the overall survival (OS) of HNSCC patients suggesting that the immune system might be targeted by these drugs to achieve clinical benefits for these patients. Despite $20-30 \%$ of HNSCC patients treated with mAbs directed against PD1 or its ligand PD-L1 have a better OS the efficacy is still limited [66]. Thus, there is an urgent need to increase the knowledge of the complex biology of this disease, in particular of immune escape mechanisms, anti-tumoral immune responses and the composition of the TME in order to improve treatment efficacy [67]. These data might help to identify features associated with responsiveness to immunotherapy and might also lead to the design of novel treatment regimens using single or multi-agent immunotherapies alone or in combination with standard therapies. Some drugs targeting these distinct mechanisms are currently in the clinical development or already approved for the treatment of HNSCC [1]. Next to targeting the PD1/PDL1 pathway, a number of novel immunotherapeutic targets are currently in preclinical studies and clinical phase I and II trials as single agent or in combination with other checkpoint molecules. These include drugs targeting LAG-3, TIM-3 and ICOS [68].

Impact of immune escape mechanisms on HNSCC patients' outcome 
Both, the direct immune escape mechanisms of tumor cells as well as the composition of the TME, have an impact of the outcome and prognosis of the HNSCC patients. Recently, tertiary lymphoid structures (TLS) with a high frequency of B cells were identified in HNSCC and found to be associated with an improved survival like in other tumor entities, whereas high frequencies of intratumoral B-lymphocytes rather indicated an adverse outcome [69] [70]. Germinal center derived $\mathrm{B}$ cells were present during disease progression of $\mathrm{HPV}^{+} \mathrm{HNSCC}$ and with a reduced frequency in HPV- HNSCC [71] [72].

High levels of TILs have been associated with improved survival in HPV- HNSCC [73]. The frequency of both $\mathrm{CD}^{+}$and $\mathrm{CD}^{+} \mathrm{T}$ cells, have been associated with an increased OS after chemoradiotherapy in $\mathrm{HPV}^{+}$and HPV- HNSCC [74] [75] [76]. In several studies, HNSCC have been shown to possess a high degree of Treg infiltration [70] [77] [78], which correlates with a favourable OS [70] [78]. This might reflect the downregulation of inflammation, which triggers the initiation of carcinogenesis [79].

In a more recent multicenter study of patients with HNSCC after post-operative chemoradiotherapy, a high CD8 TIL density in the tumor periphery, tumor stroma, and tumor cell area was predictive for improved OS [74]. In another study, only stromal TIL infiltration was associated with increased OS [80]. Concerning the clinical relevance of PD-L1 expression on the outcome of HNSCC patients, there exist controversial data. In a laryngeal HNSCC cohort, high PD-L1 expression assessed by Automated Quantitative protein Analysis (AQUA) positively correlated with disease outcome [81]. In a recent report by Yang and co-authors, PD-L1 was shown to correlate with improved progression-free survival (PFS), but not OS in patients with advanced HNSCC. As expected, patients with combined low frequency of TILs and high expression of PD-L1 were characterized by dismal survival [82]. Another retrospective analysis assessing the PD-L1 expression in a large cohort of patients demonstrated that high PD-L1 expression was the strongest predictor of worse patients' outcome independent of tumor stage and distant metastases [83]. In cancers of the oral cavity, increased PD-L1 expression has also been shown to correlate with a poor patients' survival [84]. Furthermore, TIM-3, LAG-3, IDO and CTLA4 expression was negatively correlated with OS [85].

\section{$\underline{\text { Therapeutic strategies to overcome immune escape mechanisms in HNSCC patients }}$}

So far, the OS for patients with recurrent/metastatic (R/M) disease is only 10-13 months [86] [87] [88][88, 89] when applying the current standard of care for locally recurrent disease (without 
surgical or radiation treatment options) and/or metastatic disease in the first-line setting with platinum-based double chemotherapy and cetuximab. Furthermore, second-line treatment options with cetuximab, methotrexate and taxane demonstrated a response rate between 10-13 $\%$ and median PFS of 2-3 months without any obvious improvement in OS [90] [91]. The data from Checkmate 141 and KEYNOTE-040 provided evidence for the use of single agent anti-PD1 immunotherapy for the treatment of R/M HNSCC patients with disease progression after platinum-based chemotherapy. Furthermore, the KEYNOTE-048 study using anti-PD-1 therapy in patients with R/M HNSCC who had not received prior treatment with platinum-based chemotherapy also revealed a clinical benefit suggesting the use of pembrolizumab as an appropriate fist-line treatment for PD-L1+ HNSCC [92]. In 2016, the US Food and Drug Administration (FDA) received the first immunotherapeutic approvals for the treatment of HNSCC patients with $\mathrm{iCPI}$, namely the anti-PD-1 immune checkpoint inhibitors nivolumab and pembrolizumab, for the treatment of patients with HNSCC that are refractory to platinum-based regimens. The European Commission followed in 2017 with the approval of nivolumab and shortly thereafter with the approval of pembrolizumab monotherapy for the treatment of recurrent or metastatic HNSCC in adults, whose tumors express PD-L1 with a $\geq 50 \%$ tumor proportion score and that have progressed after platinum-containing chemotherapy. In 2019, the FDA granted approval of pembrolizumab as first-line treatment for patients with metastatic or unresectable, recurrent $\mathrm{HNSCC}$, in combination with platinum and fluorouracil and of pembrolizumab as a single agent for patients with HNSCC, whose tumors express a PD-L1 combined positive score $\geq 1$. These approvals marked the first new therapies for these patients since 2006 as well as the first immunotherapeutic approvals in this disease.

However, to improve immunotherapy a much better understanding of emerging immunotherapies, including appropriate patients' selection, therapy sequence, response monitoring, adverse event management and biomarker testing, is required to guide improvements in care. In order to address these issues, the Society for Immunotherapy of Cancer (SITC) established the Cancer Immunotherapy Guideline - Head and Neck Cancer subcommittee to provide evidence-based recommendations on how to incorporate immunotherapies into practice for the treatment of patients with HNSCC [1].

In this context, insights into the complex biology of this disease in particular of the anti-tumoral immune response and the TME is urgently required. This knowledge might help to identify features associated with non-responders and responders as well as the development of resistance to the immunotherapies. Based on this information, novel treatment regimens using 
single or multi-agent immunotherapies alone or in combination with standard therapies could be developed. Next to the PD1/PD-L1 pathways, some drugs targeting these distinct mechanisms are currently in preclinical studies, in clinical phase I and II trials as single agent or in combination with other checkpoint molecules or already approved for the treatment of HNSCCs. These include antibodies directed against LAG-3, TIM-3, TIGIT and ICOS [68] [93] [94] [95]. In addition, a bifunctional fusion protein to block PD-L1 and $\beta$ receptor has been applied in a phase I trial demonstrating a clinical activity [96].

Further approaches address the natural killer antibody-dependent cell cytotoxicity and the use of NK cell therapy. Since the expression of HLA-I is reduced up to $80 \%$ compared to the surrounding healthy tissues low/neg. [30-32], NK cells might have the power to eliminate the HLA-I HNSCC cells via the missing self-recognition. However, HNSCCs express high levels of HLA-G and HLAE [40] [44], which inhibits both NK and CD8 ${ }^{+}$cells via ILT2/4 and/or NKG2A [97, 98]. Therefore, mAbs targeting NKG2A, such as monalizumab could be employed for the treatment of HNSCC by blocking the interaction with HLA-E and/or HLA-G. Indeed, a phase II study of monalizumab combined with cetuximab showed an excellent activity in previously treated HNSCC patients including some previously exposed to cetuximab [44].

\section{$\underline{\text { Conclusions }}$}

It is obvious that HNSCC are able to develop or use a multitude of mechanisms to escape T and/or NK cell-mediated immune surveillance Thus, one single strategy might be often not sufficient to mount proper immune responses, since HNSCC evade immune recognition by different approaches, which explain the limited efficacy of immune therapies in this disease. Increased insights to the immune escape mechanisms will help in the design of novel as well as optimized immune therapeutic strategies, which either have to be directed against tumor cells or revert the immune suppressive TME. 


\section{Acknowledgements}

We would like to thank Vanessa Goldhorn and Nicole Ott for excellent secretarial help.

\section{Author's contributions}

Barbara Seliger conceptualized and wrote the review, Claudia Wickenhauser was responsible for the clinical part, all authors worked on the manuscript, discussed the contents and corrected the manuscript.

\section{Conflict of interest}

The authors have no conflict of interest. 


\section{References}

1. Cohen, E.E.W., et al., The Society for Immunotherapy of Cancer consensus statement on immunotherapy for the treatment of squamous cell carcinoma of the head and neck (HNSCC). J Immunother Cancer, 2019. 7(1): p. 184.

2. Bray, F., et al., Global cancer statistics 2018: GLOBOCAN estimates of incidence and mortality worldwide for 36 cancers in 185 countries. CA Cancer J Clin, 2018. 68(6): p. 394-424.

3. Kafka, H., G.W. Burggraf, and J.A. Milliken, Electrocardiographic diagnosis of left ventricular hypertrophy in the presence of left bundle branch block: an echocardiographic study. Am J Cardiol, 1985. 55(1): p. 103-6.

4. Goldenberg, D., et al., Habitual risk factors for head and neck cancer. Otolaryngol Head Neck Surg, 2004. 131(6): p. 986-93.

5. Qian, X., et al., Heterogeneity of Head and Neck Squamous Cell Carcinoma Stem Cells. Adv Exp Med Biol, 2019. 1139: p. 23-40.

6. Hanna, G.J., et al., Defining an inflamed tumor immunophenotype in recurrent, metastatic squamous cell carcinoma of the head and neck. Oral Oncol, 2017. 67: p. 6169.

7. Muhammad, N., et al., Involvement of c-Fos in the Promotion of Cancer Stem-like Cell Properties in Head and Neck Squamous Cell Carcinoma. Clin Cancer Res, 2017. 23(12): p. 3120-3128.

8. Boshoff, C. and R. Weiss, AIDS-related malignancies. Nat Rev Cancer, 2002. 2(5): p. 373-82.

9. Burnet, F.M., The concept of immunological surveillance. Prog Exp Tumor Res, 1970. 13: p. 1-27.

10. Cervinkova, M., P. Kucerova, and J. Cizkova, Spontaneous regression of malignant melanoma - is it based on the interplay between host immune system and melanoma antigens? Anticancer Drugs, 2017. 28(8): p. 819-830.

11. Beachler, D.C., et al., Incidence and risk factors of HPV-related and HPV-unrelated Head and Neck Squamous Cell Carcinoma in HIV-infected individuals. Oral Oncol, 2014. 50(12): p. 1169-76.

12. Lei, X., et al., Immune cells within the tumor microenvironment: Biological functions and roles in cancer immunotherapy. Cancer Lett, 2020. 470: p. 126-133.

13. Schreiber, R.D., L.J. Old, and M.J. Smyth, Cancer immunoediting: integrating immunity's roles in cancer suppression and promotion. Science, 2011. 331(6024): p. 1565-70.

14. Dunn, G.P., L.J. Old, and R.D. Schreiber, The immunobiology of cancer immunosurveillance and immunoediting. Immunity, 2004. 21(2): p. 137-48.

15. Yarmarkovich, M., et al., Immunogenicity and Immune Silence in Human Cancer. Front Immunol, 2020. 11: p. 69.

16. Mittal, D., et al., New insights into cancer immunoediting and its three component phases--elimination, equilibrium and escape. Curr Opin Immunol, 2014. 27: p. 16-25.

17. Blankenstein, T., et al., The determinants of tumour immunogenicity. Nat Rev Cancer, 2012. 12(4): p. 307-13.

18. Galon, J., et al., The continuum of cancer immunosurveillance: prognostic, predictive, and mechanistic signatures. Immunity, 2013. 39(1): p. 11-26.

19. Vigneron, N., Human Tumor Antigens and Cancer Immunotherapy. Biomed Res Int, 2015. 2015: p. 948501.

20. Coulie, P.G., et al., Tumour antigens recognized by T lymphocytes: at the core of cancer immunotherapy. Nat Rev Cancer, 2014. 14(2): p. 135-46.

21. Pamer, E. and P. Cresswell, Mechanisms of MHC class I--restricted antigen processing. Annu Rev Immunol, 1998. 16: p. 323-58. 
22. Panter, M.S., et al., Dynamics of major histocompatibility complex class I association with the human peptide-loading complex. J Biol Chem, 2012. 287(37): p. 31172-84.

23. Harjunpaa, H., et al., Cell Adhesion Molecules and Their Roles and Regulation in the Immune and Tumor Microenvironment. Front Immunol, 2019. 10: p. 1078.

24. Dubey, C., M. Croft, and S.L. Swain, Costimulatory requirements of naive CD4+ $T$ cells. ICAM-1 or B7-1 can costimulate naive CD4 T cell activation but both are required for optimum response. J Immunol, 1995. 155(1): p. 45-57.

25. Labani-Motlagh, A., M. Ashja-Mahdavi, and A. Loskog, The Tumor Microenvironment: A Milieu Hindering and Obstructing Antitumor Immune Responses. Front Immunol, 2020. 11: p. 940.

26. Maleki Vareki, S., High and low mutational burden tumors versus immunologically hot and cold tumors and response to immune checkpoint inhibitors. J Immunother Cancer, 2018. 6(1): p. 157.

27. Allegrezza, M.J. and J.R. Conejo-Garcia, Targeted Therapy and Immunosuppression in the Tumor Microenvironment. Trends Cancer, 2017. 3(1): p. 19-27.

28. Munn, D.H. and V. Bronte, Immune suppressive mechanisms in the tumor microenvironment. Curr Opin Immunol, 2016. 39: p. 1-6.

29. Erra Diaz, F., E. Dantas, and J. Geffner, Unravelling the Interplay between Extracellular Acidosis and Immune Cells. Mediators Inflamm, 2018. 2018: p. 1218297.

30. Vora, A.R., et al., An immunohistochemical study of altered immunomodulatory molecule expression in head and neck squamous cell carcinoma. Br J Cancer, 1997. 76(7): p. 836-44.

31. Yuan, R.H., et al., Opposite roles of human pancreatitis-associated protein and REG1A expression in hepatocellular carcinoma: association of pancreatitis-associated protein expression with low-stage hepatocellular carcinoma, beta-catenin mutation, and favorable prognosis. Clin Cancer Res, 2005. 11(7): p. 2568-75.

32. Feenstra, M., et al., HLA class I expression and chromosomal deletions at $6 p$ and $15 q$ in head and neck squamous cell carcinomas. Tissue Antigens, 1999. 54(3): p. 235-45.

33. Meissner, M., et al., Defects in the human leukocyte antigen class I antigen processing machinery in head and neck squamous cell carcinoma: association with clinical outcome. Clin Cancer Res, 2005. 11(7): p. 2552-60.

34. Cioni, B., et al., HLA class II expression on tumor cells and low numbers of tumorassociated macrophages predict clinical outcome in oropharyngeal cancer. Head Neck, 2019. 41(2): p. 463-478.

35. Feenstra, M., et al., Microsatellite analysis of microdissected tumor cells and $6 p$ high density microsatellite analysis in head and neck squamous cell carcinomas with downregulated human leukocyte antigen class I expression. Lab Invest, 2000. 80(3): p. 40514.

36. Lopez-Albaitero, A., et al., Role of antigen-processing machinery in the in vitro resistance of squamous cell carcinoma of the head and neck cells to recognition by CTL. $\mathrm{J}$ Immunol, 2006. 176(6): p. 3402-9.

37. Ogino, T., et al., HLA class I antigen down-regulation in primary laryngeal squamous cell carcinoma lesions as a poor prognostic marker. Cancer Res, 2006. 66(18): p. 9281-9.

38. Leibowitz, M.S., et al., Deficiency of activated STAT1 in head and neck cancer cells mediates TAP1-dependent escape from cytotoxic T lymphocytes. Cancer Immunol Immunother, 2011. 60(4): p. 525-35.

39. Moss, W.J., Is It Time to Rethink the Approach to Internal Nasal Valve Stenosis? JAMA Otolaryngol Head Neck Surg, 2019. 145(2): p. 105-106.

40. Shen, X., et al., Correlation between human leukocyte antigen-G expression and clinical parameters in oral squamous cell carcinoma. Indian J Cancer, 2018. 55(4): p. 340-343. 
41. Goncalves, A.S., et al., Immunosuppressive mediators of oral squamous cell carcinoma in tumour samples and saliva. Hum Immunol, 2015. 76(1): p. 52-8.

42. Sarmah, N., M.N. Baruah, and S. Baruah, Immune Modulation in HLA-G Expressing Head and Neck Squamous Cell Carcinoma in Relation to Human Papilloma Virus Positivity: A Study From Northeast India. Front Oncol, 2019. 9: p. 58.

43. Goncalves, A.S., et al., The clinicopathologic significance of the expression of HLA-G in oral squamous cell carcinoma. Oral Surg Oral Med Oral Pathol Oral Radiol, 2014. 117(3): p. 361-8.

44. Andre, P., et al., Anti-NKG2A mAb Is a Checkpoint Inhibitor that Promotes Anti-tumor Immunity by Unleashing Both T and NK Cells. Cell, 2018. 175(7): p. 1731-1743 e13.

45. Schneider, S., et al., $P D-1$ and $P D-L 1$ expression in HNSCC primary cancer and related lymph node metastasis - impact on clinical outcome. Histopathology, 2018. 73(4): p. 573-584.

46. Takahashi, H., et al., Clinical and Biological Significance of PD-L1 Expression Within the Tumor Microenvironment of Oral Squamous Cell Carcinoma. Anticancer Res, 2019. 39(6): p. 3039-3046.

47. Wang, H.C., L.P. Chan, and S.F. Cho, Targeting the Immune Microenvironment in the Treatment of Head and Neck Squamous Cell Carcinoma. Front Oncol, 2019. 9: p. 1084.

48. Yamamoto, M., H. Inohara, and T. Nakagawa, Targeting metabolic pathways for head and neck cancers therapeutics. Cancer Metastasis Rev, 2017. 36(3): p. 503-514.

49. Zabeida, A., et al., Reevaluating immunization delays after red blood cell transfusion. Transfusion, 2019. 59(9): p. 2806-2811.

50. Curry, J.M., et al., Cancer metabolism, stemness and tumor recurrence: MCT1 and MCT 4 are functional biomarkers of metabolic symbiosis in head and neck cancer. Cell Cycle, 2013. 12(9): p. 1371-84.

51. Samanta, D. and G.L. Semenza, Metabolic adaptation of cancer and immune cells mediated by hypoxia-inducible factors. Biochim Biophys Acta Rev Cancer, 2018. 1870(1): p. 15-22.

52. Boothby, M. and R.C. Rickert, Metabolic Regulation of the Immune Humoral Response. Immunity, 2017. 46(5): p. 743-755.

53. Montler, R., et al., OX40, PD-1 and CTLA-4 are selectively expressed on tumorinfiltrating T cells in head and neck cancer. Clin Transl Immunology, 2016. 5(4): p. e70.

54. Kumar, A.T., et al., Prognostic Significance of Tumor-Associated Macrophage Content in Head and Neck Squamous Cell Carcinoma: A Meta-Analysis. Front Oncol, 2019. 9: p. 656.

55. Chambers, S.E., et al., The role of immune-related myeloid cells in angiogenesis. Immunobiology, 2013. 218(11): p. 1370-5.

56. Aguilar-Cazares, D., et al., Contribution of Angiogenesis to Inflammation and Cancer. Front Oncol, 2019. 9: p. 1399.

57. Schmitz, S., et al., Cetuximab promotes epithelial to mesenchymal transition and cancer associated fibroblasts in patients with head and neck cancer. Oncotarget, 2015. 6(33): p. 34288-99.

58. Kumar, D., et al., Cancer-Associated Fibroblasts Drive Glycolysis in a Targetable Signaling Loop Implicated in Head and Neck Squamous Cell Carcinoma Progression. Cancer Res, 2018. 78(14): p. 3769-3782.

59. Chai, A.W.Y., K.P. Lim, and S.C. Cheong, Translational genomics and recent advances in oral squamous cell carcinoma. Semin Cancer Biol, 2020. 61: p. 71-83.

60. Su, S.C., et al., Exome Sequencing of Oral Squamous Cell Carcinoma Reveals Molecular Subgroups and Novel Therapeutic Opportunities. Theranostics, 2017. 7(5): p. 1088-1099. 
61. Feng, Z., et al., Multiparametric immune profiling in HPV-oral squamous cell cancer. JCl Insight, 2017. 2(14).

62. Gluck, C., et al., Molecular dissection of the oncogenic role of ETS1 in the mesenchymal subtypes of head and neck squamous cell carcinoma. PLoS Genet, 2019. 15(7): p. e1008250.

63. Liu, C., et al., Characterization of Alternative Splicing Events in HPV-Negative Head and Neck Squamous Cell Carcinoma Identifies an Oncogenic DOCK5 Variant. Clin Cancer Res, 2018. 24(20): p. 5123-5132.

64. Canning, M., et al., Heterogeneity of the Head and Neck Squamous Cell Carcinoma Immune Landscape and Its Impact on Immunotherapy. Front Cell Dev Biol, 2019. 7: p. 52.

65. Jie, H.B., et al., Increased PD-1(+) and TIM-3(+) TILs during Cetuximab Therapy Inversely Correlate with Response in Head and Neck Cancer Patients. Cancer Immunol Res, 2017. 5(5): p. 408-416.

66. Zolkind, P. and R. Uppaluri, Checkpoint immunotherapy in head and neck cancers. Cancer Metastasis Rev, 2017. 36(3): p. 475-489.

67. Moy, J.D., J.M. Moskovitz, and R.L. Ferris, Biological mechanisms of immune escape and implications for immunotherapy in head and neck squamous cell carcinoma. Eur $\mathrm{J}$ Cancer, 2017. 76: p. 152-166.

68. Wu, L., et al., Blockade of TIGIT/CD155 Signaling Reverses T-cell Exhaustion and Enhances Antitumor Capability in Head and Neck Squamous Cell Carcinoma. Cancer Immunol Res, 2019. 7(10): p. 1700-1713.

69. Woo, J.R., et al., Tumor infiltrating B-cells are increased in prostate cancer tissue. J Transl Med, 2014. 12: p. 30.

70. Ou, D., et al., Clinical relevance of tumor infiltrating lymphocytes, PD-L1 expression and correlation with HPV/p16 in head and neck cancer treated with bio- or chemoradiotherapy. Oncoimmunology, 2017. 6(9): p. e1341030.

71. Cillo, A.R., et al., Immune Landscape of Viral- and Carcinogen-Driven Head and Neck Cancer. Immunity, 2020. 52(1): p. 183-199 e9.

72. Shi, J.Y., et al., Margin-infiltrating CD20(+) B cells display an atypical memory phenotype and correlate with favorable prognosis in hepatocellular carcinoma. Clin Cancer Res, 2013. 19(21): p. 5994-6005.

73. Ward, M.J., et al., Tumour-infiltrating lymphocytes predict for outcome in HPV-positive oropharyngeal cancer. Br J Cancer, 2014. 110(2): p. 489-500.

74. Balermpas, P., et al., Tumour-infiltrating lymphocytes predict response to definitive chemoradiotherapy in head and neck cancer. Br J Cancer, 2014. 110(2): p. 501-9.

75. Balermpas, P., et al., CD8+ tumour-infiltrating lymphocytes in relation to HPV status and clinical outcome in patients with head and neck cancer after postoperative chemoradiotherapy: A multicentre study of the German cancer consortium radiation oncology group (DKTK-ROG). Int J Cancer, 2016. 138(1): p. 171-81.

76. Fridman, W.H., et al., The immune contexture in cancer prognosis and treatment. Nat Rev Clin Oncol, 2017. 14(12): p. 717-734.

77. Mandal, R., et al., The head and neck cancer immune landscape and its immunotherapeutic implications. JCI Insight, 2016. 1(17): p. e89829.

78. Badoual, C., et al., Prognostic value of tumor-infiltrating CD4+ T-cell subpopulations in head and neck cancers. Clin Cancer Res, 2006. 12(2): p. 465-72.

79. Erdman, S.E., et al., Unifying roles for regulatory $T$ cells and inflammation in cancer. Int J Cancer, 2010. 126(7): p. 1651-65.

80. Oguejiofor, K., et al., Stromal infiltration of CD8 T cells is associated with improved clinical outcome in HPV-positive oropharyngeal squamous carcinoma. $\mathrm{Br} \mathrm{J}$ Cancer, 2015. 113(6): p. 886-93. 
81. Vassilakopoulou, M., et al., Evaluation of PD-L1 Expression and Associated TumorInfiltrating Lymphocytes in Laryngeal Squamous Cell Carcinoma. Clin Cancer Res, 2016. 22(3): p. 704-13.

82. Yang, W.F., et al., The prognostic role of PD-L1 expression for survival in head and neck squamous cell carcinoma: A systematic review and meta-analysis. Oral Oncol, 2018. 86: p. 81-90.

83. Muller, T., et al., PD-L1: a novel prognostic biomarker in head and neck squamous cell carcinoma. Oncotarget, 2017. 8(32): p. 52889-52900.

84. Lin, Y.M., et al., High PD-L1 Expression Correlates with Metastasis and Poor Prognosis in Oral Squamous Cell Carcinoma. PLoS One, 2015. 10(11): p. e0142656.

85. Wang, $\mathrm{H}$., et al., Altered expression of TIM-3, LAG-3, IDO, PD-L1, and CTLA-4 during nimotuzumab therapy correlates with responses and prognosis of oral squamous cell carcinoma patients. J Oral Pathol Med, 2019. 48(8): p. 669-676.

86. Brockstein, B., et al., Patterns of failure, prognostic factors and survival in locoregionally advanced head and neck cancer treated with concomitant chemoradiotherapy: a 9-year, 337-patient, multi-institutional experience. Ann Oncol, 2004. 15(8): p. 1179-86.

87. Bourhis, J., et al., Individual patients' data meta-analyses in head and neck cancer. Curr Opin Oncol, 2007. 19(3): p. 188-94.

88. Posner, M.R., et al., Cisplatin and fluorouracil alone or with docetaxel in head and neck cancer. N Engl J Med, 2007. 357(17): p. 1705-15.

89. de Bree, R., et al., Screening for distant metastases in patients with head and neck cancer. Laryngoscope, 2000. 110(3 Pt 1): p. 397-401.

90. Leon, X., et al., A retrospective analysis of the outcome of patients with recurrent and/or metastatic squamous cell carcinoma of the head and neck refractory to a platinumbased chemotherapy. Clin Oncol (R Coll Radiol), 2005. 17(6): p. 418-24.

91. Fakhry, C., et al., Improved survival of patients with human papillomavirus-positive head and neck squamous cell carcinoma in a prospective clinical trial. J Natl Cancer Inst, 2008. 100(4): p. 261-9.

92. Burtness, B., et al., Pembrolizumab alone or with chemotherapy versus cetuximab with chemotherapy for recurrent or metastatic squamous cell carcinoma of the head and neck (KEYNOTE-048): a randomised, open-label, phase 3 study. Lancet, 2019. 394(10212): p. 1915-1928.

93. Suzuki, S., et al., Immune-checkpoint molecules on regulatory T-cells as a potential therapeutic target in head and neck squamous cell cancers. Cancer Sci, 2020. 111(6): p. 1943-1957.

94. de Vos, L., et al., The landscape of CD28, CD80, CD86, CTLA4, and ICOS DNA methylation in head and neck squamous cell carcinomas. Epigenetics, 2020: p. 1-18.

95. Liu, J.F., et al., Blockade of TIM3 relieves immunosuppression through reducing regulatory $T$ cells in head and neck cancer. J Exp Clin Cancer Res, 2018. 37(1): p. 44.

96. Doi, T., et al., Phase I Study of the Bifunctional Fusion Protein Bintrafusp Alfa in Asian Patients with Advanced Solid Tumors, Including a Hepatocellular Carcinoma SafetyAssessment Cohort. Oncologist, 2020.

97. Ho, G.T., et al., NKG2A/CD94 Is a New Immune Receptor for HLA-G and Distinguishes Amino Acid Differences in the HLA-G Heavy Chain. Int J Mol Sci, 2020. 21(12).

98. Petrie, E.J., et al., CD94-NKG2A recognition of human leukocyte antigen (HLA)-E bound to an HLA class I leader sequence. J Exp Med, 2008. 205(3): p. 725-35. 